\title{
Analysis and Detection of Nephrolithiasis using Imaging Techniques
}

\author{
Smiti Tripathy ${ }^{1}$, Sivakumar R ${ }^{1}$, Simran Nair ${ }^{1}$, Inbamalar TM² \\ ${ }^{1}$ School of Electronics Engineering, Vellore Institute of Technology, Vellore, Tamilnadu, India \\ ${ }^{2} \mathrm{RMK}$ College of Engineering and Technology, Tamilnadu, India \\ *rsivakumar@vit.ac.in
}

Received: January 6, 2020. Revised: February 3, 2021. Accepted: February 12, 2021.

Published: February 17, 2021.

Received: August 22, 2020. Revised: January 28, 2021. Accepted: February 12, 2021. Published: February 17, 2021.

\begin{abstract}
Nephrolithiasis (kidney stone) is a disease which affects $7 \%$ of females and $11 \%$ of males at some stage in their life. Early identification of Nephrolithiasis is necessary to avoid complications. Imaging techniques form the basis for the detection of kidney stones and aid in locating the position, size, and the number of stones present in the renal structure. This paper reports an extensive analysis of recent trends in the detection of Nephrolithiasis using Imaging techniques. Since Computed Tomography (CT) and ultrasound imaging are commonly used in the medical field, analysis of both the methods is considered in this paper. The detailed study on various methodologies and algorithms that have been adopted on CT and ultrasound images in recent years in locating kidney stones, finding the exact size of the stones based on pixel count, enhancing image quality, obtaining better de-speckling, faster segmentation, and pre-processing of the renal images has been carried out. Based on the analysis, an artificial intelligence-based approach is proposed that will aid the medical practitioner for faster, accurate detection of Nephrolithiasis and a technique to reduce the exposure of radiation in Computed Tomography Imaging. Further, it is concluded that ultrasound techniques can be employed subsequently for preliminary diagnosis through CT if the medical practitioner recommends.
\end{abstract}

Keywords: Computed Tomography, Kidney stone, Nephrolithiasis, Ultrasound Imaging

\section{INTRODUCTION}

Kidneys are the filtering organs of the body. They are located at the sides of the abdomen responsible for the purification of blood through nephrons, and they produce urine (extra water containing urea, ions, toxic ammonia, carbon dioxide, sodium) as a waste by-product. The presence of less fluid in the body, the ions, and the other waste product deposits hard masses in the kidney known as kidney stones. They can be present anywhere in the urinary tract. Nephrolithiasis or Renal calculus is the scientific term for kidney stone. In the medical field, this condition is called Nephrolithiasis or urinary stones. Usually, stones originate from kidneys under the age group of $20-50$ years or in premature infants. Though anyone can face kidney stone problem, there are a few conditions which increase the chance of stone development such as intake of insufficient water, consumption of food rich in sodium, sugar, and protein, genetic (presence of stones in the family) factors, overweight, intake of water-pills (diuretic) or calcium based antacids, gastro-intestinal or gastric-bypass surgery, and the presence of polycystic kidney disease. A sample image of the kidney with stones is shown in figure 1 .

There are different types of kidney stones based on the significant constituents they are formed from,

1) Calcium oxalate stones - These types of stones are formed when urine contains a high amount of calcium, oxalate, and low levels of citrate. The majority of the renal stone is due to this calcium oxalate [28]. Diet rich in oxalates such as chocolates, nuts, and spinach are responsible for stone formation.

2) Calcium phosphate stones - These types of stones are produced due to the malfunctioning of the Urinary system, often present with calcium oxalate stones.

3) Struvite stones - These are the types of stones formed due to infections in the urinary tract. They grow fast and occupy a large area. These stones are sometimes called as the stag horn calculi when they form hefty enough [29]. These are most commonly found in women.

4) Uric acid stones - These are formed due to insufficient intake of water, a diet rich in animal protein, gout (a type of arthritis developed due to high amount of uric acid in the blood), or can develop after chemotherapy most commonly found in men.

5) Cystine stones - These are formed due to the high amount of cystine (amino acid) present in urine. Most commonly, it is a hereditary disorder called cystinuria, which can result in stone formation at any part of the urinary tract (kidney, bladder, and urethra). 


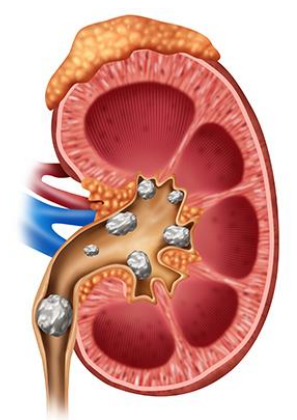

Fig1: Image of stones present in the kidney

(Source: Medscape article, 2018)

Stones in kidneys generally do not give rise to warning signs and may pass undiagnosed. On the other hand, if a stone obstructs the stream of urine out of the kidney, it may lead to severe pain. Pain in the abdomen or at the sides is the main indication of kidney stones. Symptoms like - fever, chills, vomiting, nausea, blood in urine, badodour, and reduction in the amount of urine and frequent urge for urination are other most common symptoms of kidney stones, which should not be ignored. When the size of the stone is small, no symptoms or pain would be visible; however, as the mass of the stone increases pain in the abdomen or groin area develops. Proper hydration (intake of water), more consumption of citrate juices, oxalate food, and low intake of animal proteins are a few ways to prevent the formation of kidney stones. Approximately 11 percent of male and 6 percent of the female have kidney stones at least once all through their life span. If kidney stones are not taken care of, they can cause haematuria, kidney infections and the loss of kidney function.

Detection of kidney stones can be done in several ways: urinalysis (test for blood, white cell, bacteria, and crystals), BUN (blood urea nitrogen) and creatinine to diagnose urinary tract function, test for calcium, electrolytes, and phosphorus in the blood stream. There are also other detection methods available for kidney stones such as Ultrasound Imaging of kidney, X-ray, and Computerized Tomography (CT) of the abdomen, Magnetic resonance imaging (MRI) of a kidney, and abdomen, and Intravenous Pyelogram (IVP).

In this paper, various imaging techniques have been taken into consideration. A review of the imaging techniques and their performance analysis are also presented. A detailed analysis of various algorithms adopted in Ultrasound and CT images for kidney stone is discussed. Furthermore artificial intelligence and deep learning techniques are presented in section III. The proposed AI based approach for $\mathrm{f}$ detection of renal stone is presented in the Section IV.

\section{REVIEW OF IMAGING TECHNIQUES}

Imaging tests have been used to show kidney stones in the human urinary tract. Ultrasound Imaging, X-ray Imaging, Computerized Tomography, Magnetic resonance imaging techniques in use in the medical field for the diagnosis of Nephrolithiasis.

\section{A. Ultrasound Imaging}

Ultrasound technology uses sound waves to create a picture of kidneys and bladder. Primarily ultrasound imaging has been in use as a diagnostic technique. Due to later advancements, it is also used for the treatment of kidney stones and therapy guidance aid [1]. Ultrasound is a non-invasive test and it is fast and simple to carry out. These machines are smaller, portable, and are capable of real-time imaging. Hence urologists use ultrasound to locate stone as well as the removal of stones. Moreover, for paediatric and pregnant patients, ultrasound is widely accepted as the diagnostic tool.

\section{B. X rays}

In standard X-rays, a beam of energy is aimed at the body part being studied. A plate behind the body part captures variations of the energy beam after it passes through the skin, bone, muscle, and other tissues. Nephrolithiasis in the urinary tract can be detected using abdominal x-rays. It can show the location of Kidney stones in the urinary tract. Not all stones are visible on abdominal x-ray.

\section{Kidney, Ureter, Bladder Radiography and Fluoroscopy}

Normally X-ray units consist of both radiographic and fluoroscopic facilities. It contains a high voltage power supply, an X-ray tube, a collimating device, and an X-ray detector or film. Fluoroscopy technique makes use of Xrays to acquire instantaneous moving images of the interior of the body. It includes an electronic image intensifier and an image display system. In Kidney, ureter, bladder (KUB) plain film radiography and fluoroscopy, photons produced by a single energy source pass through tissues. This technique uses the same fundamental concepts as CT but in a single plane. Advantages of KUB radiography include relatively low ionizing radiation exposure compared with $\mathrm{CT}$ and low cost compared to ultrasonography. However, KUB radiography views stones at one angle and, therefore, accuracy are decreased leading to reduced sensitivity and specificity and, consequently, limiting its utility.

\section{Intravenous Pyelogram (IVP)}

IVP or intravenous urogram is a radiological procedure that uses an injection of contrast material to detect Nephrolithiasis. Contrast materials may cause undesirable allergic effects in some people. IVP tests are rarely used in infants and children. Instead, ultrasound tests are advised for paediatric patients 


\section{E. Computerized Tomography}

CT scans use is amalgamation of x-rays and computer technology to generate images of the urinary tract. In computed tomography, the X-ray beam moves in a circle around the body. The X-ray information is sent to a computer that interprets the X-ray data and displays it in two-dimensions (2D) on a monitor. CT scans may be done with or without "contrast." Contrast refers to a substance taken by mouth or injected into an intravenous (IV) line that causes the particular organ or tissue under study to be seen more clearly. CT scans of the kidneys can provide more detailed information about the kidneys than standard kidney, ureter, and bladder (KUB) X-rays. CT scans can show the size and location of a kidney stone if the stone is blocking the urinary tract, and can also show conditions that may have caused the kidney stone to form.

\section{F. Magnetic resonance Imaging}

MRI uses a magnetic field to align the patient's free water protons along a magnetic field axis. A radiofrequency antenna, referred to as a coil, is placed over the area to be imaged and releases pulses of energy that disrupt the alignment of the protons. When the pulses stop, protons release energy as they realign with the magnetic field this released energy can be captured as an image. The sensitivity of MRI is higher than that of ultrasonography and KUB radiography but less than that of CT, as stones are less easily visible when using MRI. A major advantage of MRI is the ability to provide $3 \mathrm{D}$ imaging without radiation. Unfortunately, the drawbacks of MRI prevent it from widespread use in stone imaging. In general, MRI costs about three times more than a CT scan and has lower accuracy and much longer image acquisition times. MRI is probably the most appropriately used as an adjunctive to ultrasonography in patients that are pregnant.

\section{G. Summary}

High-speed or dual energy computerized tomography (CT) may reveal even tiny stones. Simple abdominal Xrays are used less frequently because this kind of imaging test can miss small kidney stones. During a CT scan, the patient is briefly exposed to ionizing radiation. The amount of radiation is greater than one would get during a plain X-ray because the CT scan gathers more detailed information. The low doses of radiation used in CT scans have not been shown to cause long-term harm, although, at much higher doses, there may be a small increase in the risk of cancer. MRI scans which do not use radiation are not typically used to evaluate kidney stones. But, it helps to safely diagnose kidney stones in pregnant women.

\section{ANALYSIS OF TECHNIQUES FOR NEPHROLITHIASIS DETECTION}

In the following sections, various algorithms and techniques that have been adopted for kidney stone detection are discussed. The general steps in the image processing algorithms used in kidney stone detection are explained with a block diagram as shown in figure 2 .

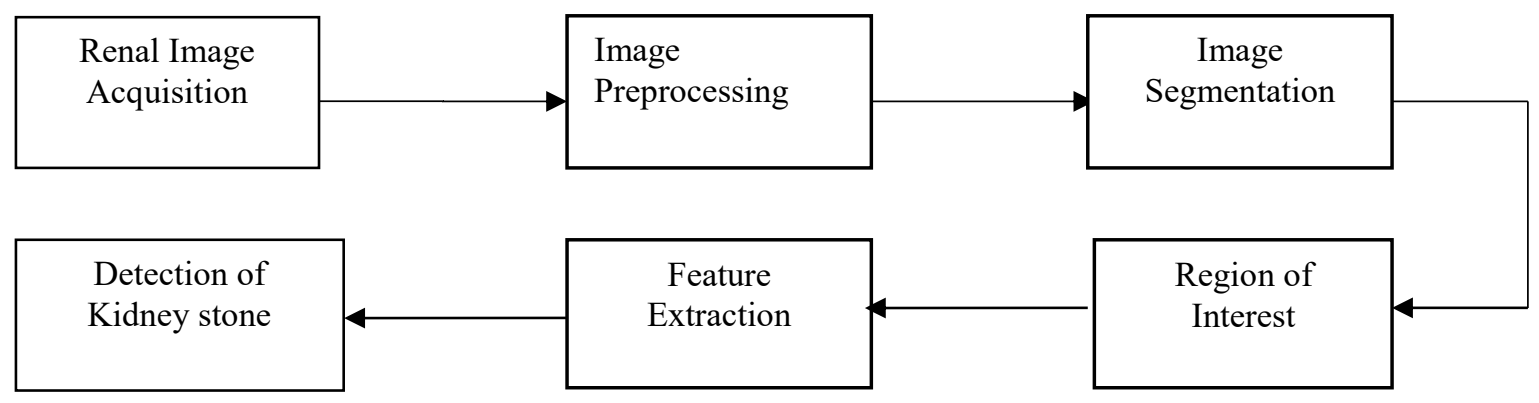

Fig2: Illustration of steps in kidney stone detection using Ultrasound Imaging

\section{A. Analysis of Kidney Stone Detection on Ultrasound Images}

Ultrasound is the diagnostic imaging technique which has several pros such as no exposure to the ionizing radiation, it's is a non-invasive procedure, that uses a device called transducer which is rubbed against the patient's skin area of examination, provides a clear detailed image of the soft tissues and is cost-effective. The ultrasonic image properties of body tissues determine the characteristics of ultrasound image. The two important parameters of the ultrasound imaging is the attenuation and the velocity of propagation that decides with how much frequency the body tissues can be imaged.It is one of the techniques used for kidney stone detection where ultrasound image acquired is processed through image processing algorithms. The diagnostic ability is reduced by speckle noise in the Ultrasound images. The difference in travelling path of the coherent acoustic waves adds up interference thereby results in introduction of speckle noise in the ultrasound image [22].Therefore; speckle noise reduction is the first pre-processing step to improve the Ultrasound image quality. To remove the speckle noise, the following filtering techniques can be utilized:

- Wavelet Filter: It provides better accuracy even in the presence of noise in the image, but this filter never raises SNR (Signal to noise ratio) high.

- Lee Filter: This is a special filter, widely used in medical Ultrasound imaging This filter reduces speckle noise by employing minimum mean squared error approach 
- Diffusion Filter: It is a non-linear filter, which utilizes a coefficient of variation to enhance the contrast in the image along with the reduction of the speckle noise.

- Bilateral Filter: It is a non-linear and noniterative filter, considered as a robust method for smoothing of noisy images and the preservation of edges. The basic concept of this filtering technique is that it replaces the noisy pixel of the image with weighted values, which depends on the photometric and the geometric distance.

After pre-processing of the ultrasound image, comes the segmentation of the image; the image segmentation can be carried out using Level set segmentation methods. Apart from Level Set segmentation methods, several other Segmentation methodologies which can be implemented on the Ultrasound image, the following lists some of the significant Segmentation methods:

- ESRANFIS Method: Effective Segmentation of Renal calculi Adaptive Neuro-Fuzzy Inference
System (ESRANFIS) method detects the renal calculi with the use of Adaptive Neuro-Fuzzy Inference System (ANFIS).This technique is utilized in two phases. In the first phase, the normal and the affected kidney are classified; in the second phase of the segmentation, the kidney stone areas are found in the classified image (Figure 3).

- RICS Method: Region Indicator with Contour Segmentation (RICS) method involves five major steps. Computation of renal calculi region parameters is carried out in the first and second steps. Histogram equalization is performed for contrast enhancement of the image, and then kmean clustering selects the most desired pixel value. The most desired pixel value is utilized to locate the stone exactly in the renal image. In the final step, pixel matching and thresholding process are performed to locate the calculi. The RICS method of segmentation shows high efficiency with the minimized error.

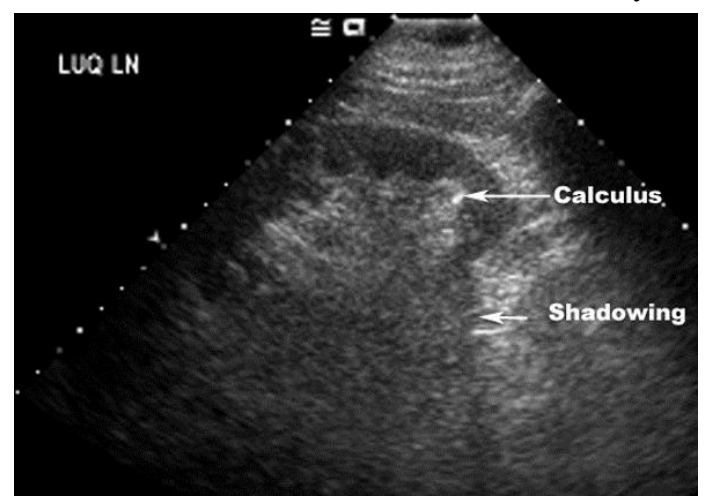

Fig3: Ultrasound image showing renal calculi.

An imaging technique for early diagnosis of Nephrolithiasis has been developed by Tamilselvi and P Thangaraj (2011) [2]. Here improved Seeded Region Growing (SRG) technique has been used for segmentation and classification. This method depends on image granularity features and the detection procedure is carried out based on intensity threshold disparities that are acquired from the segmented portions of the image. Bryan Cunitz et al (2014) proposed an improved method for Nephrolithiasis detection technique using Doppler imaging [3]. Here Doppler output parameters have been optimized in vitro to improve the overall performance of 'twinkling artefact' (TA) which a kidney stone exhibits under Color Doppler ultrasound. This method exhibits improved SNR. Viswanath K and Gunasundari R (2015) have proposed a model to detect Nephrolithiasis by Reaction-diffusion level set segmentation [4]. For implementation, Xilinx System generator on FPGA is used. Here, the ultrasound image is preprocessed to avoid speckle noise. Then smoothing by using Gabor filtering is followed by Histogram equalization. Multilayer Perceptron (MLP) and Back Propagation have been employed to categorize the form of stone.
PallaviVaish et al (2016) have developed a scheme that detects malfunctions in kidneys. Smartphone acquires the image data from

Ultrasound scanners for performing Computer-aided diagnosis and generating results [5]. Viola-Jones algorithm, texture feature extraction and Support Vector Machine (SVM) classifier are employed for automatically detecting the abnormality in the kidney. JyotiVerma et al (2017) have designed an algorithm for recognizing Nephrolithiasis using Kth Nearest Neighbour (KNN) and SVM techniques [6]. Here Image enhancement is performed using the Median filter. Gaussian and Median filters are also applied for filtering besides Un-sharp masking for sharpening. Subsequently, erosion and dilation are carried out. This is followed by entropy-based segmentation for locating the region of interest. Further processing by KNN and SVM techniques results in the final outputs

S. M. K. Chaitanya and P. Rajesh Kumar (2019) employedgrey-scale conversion for pre-processing followed by region-of-interest generation. Followed by feature extraction by Gabor wavelet followed by Cuckoo Search Artificial Neural Network (ANN) algorithm for optimization [7].To compare the performance of various methods, the various 
performance metrics for the existing methods have

been tabulated in table 1 .

\begin{tabular}{|c|c|c|c|c|}
\hline Year & Authors & Methods & Accuracy & Specificity \\
\hline 2014 & $\begin{array}{c}\text { Viswanath K } \\
\text { \&unasundari R }\end{array}$ & $\begin{array}{c}\text { Multilayer Perceptron, Back Propagation } \\
\text { ANN }\end{array}$ & $98.8 \%$ & - \\
\hline 2016 & PallaviVaish et al & Viola-Jones algorithm, SVM Classifier & $90.91 \%$ & - \\
\hline 2017 & JyotiVerma et al & KNN, SVM & $85 \%$ & - \\
\hline 2019 & $\begin{array}{c}\text { S. M. K. Chaitanya, P. } \\
\text { Rajesh Kumar }\end{array}$ & $\begin{array}{c}\text { Cuckoo Search (CS) and } \\
\text { Artificial Neural Network (ANN) }\end{array}$ & 94 & 100 \\
\hline
\end{tabular}

Table1: Performance metrics for Ultrasound Imaging

\section{B. Analysis of Nephrolithiasis Detection on CT Images}

CT is a technique that uses $\mathrm{x}$-ray equipment to create a highly detailed scan of the internal areas of the body. In recent years, the Computed Tomography imaging techniques have been used for Nephrolithiasis detection. Several types of studies have been carried out on obtaining better CT image quality; this section provides a detailed analysis of the filtering techniques and algorithms that have been implemented on CT images.

D.Y. Kim \& J.W. Park (2004) has applied a Gray level threshold method for the segmentation of the CT kidney image along with average and standard deviations to detect kidney tumor [8]. J. Kolomaznık et al. (2010) presented an algorithm for fast segmentation of kidneys in CT images [9]. Three-Dimensional segmentation on parametric snakes also called active contour model is implemented in volume slices to define the organ's volume that is the kidney. Wu Zhou and YaoqinXie (2013) have been using the active contour model in the segmentation of volumetric medical images [10]. The snake model algorithm for segmentation of medical image has been effective as almost all medical images are in 3-dimensional form. Regions are obtained in stacks to form volumetric region after images slices are segmented individually. Jianfei Liu et al. (2014) have developed a total variation (TV) flow methodology to minimize image noise in the renal structure to maintain the characteristic appearance of renal calculi, as image noise is the main challenge that hinders accurate Nephrolithiasis detection [11].

Maximally stable external regions (MSER) features were used to identify kidney stones. MSER is appropriate to detect kidney stones as it exploits extreme image values to detect image blobs. The texture and shape features are computed and are imported to support vector machines for calculus classification. SamanEbrahimi et al. (2015) worked on Kidney urine belly-CT (KUB-CT) where KUB is an imaging modality that can improve Nephrolithiasis screening and diagnosis [12]. The image processing techniques that are employed in the program, namely, Gamma Adjustment, Image Segmentation, Binarization,
Boolean operation, morphological operation, and localization improve the efficiency in the program. Sujata Navratnam et al (2016) have worked on enhanced seed pixel region growing segmentation and ANN classification which accurately distinguishes the presence and absence of renal calculi [13]. The system uses texture features which compute the pixels in each slice of the ultrasound image. A set of seeds and the image are taken as the input in the automatic region algorithm. The region of the kidney to be segmented is marked by seeds which are taken as the input. The output of the segmentation depends on the selection of seeds. The next step is feature extraction. Feature space can be intensity, texture, and shape. The feature extraction process depends on the usage of classifiers.

Prema T.Akkasaligar et al. (2017) have designed a model for Kidney stone detection in Computed Tomography (CT) images [14]. This method uses Level set segmentation where the input images are preprocessed and the region of interest is segmented. Fuzzy C-means clustering is applied to this image to obtain cluster centers. This is then segmented using Level set segmentation which is then analyzed to detect stone size and location. NilarThein et al (2018) have proposed an Image preprocessing method for the segmentation of kidney stones in CT scan images [15]. Here three thresholding algorithms that are based on intensity, size and location are applied for removing unwanted regions in $\mathrm{CT}$ images. $30 \mathrm{CT}$ scan images of the digitized transverse abdomen from the patients with kidney stones are included for validation and statistical analysis. This algorithm provided experimental results with a sensitivity of $95.24 \%$.

Stalina $\mathrm{S}$ et al (2018) designed an algorithm for detecting kidney stones on CT images using Image Processing [16]. The image is preprocessed using filters to smoothen and remove noise. It is followed by Image enhancement with Power-law transformation. The thresholding technique is applied for Image segmentation and thus kidney stones are detected.To compare the performance of various methods, the various performance metrics for the existing methods have been tabulated and are listed in table 2 . 
Researchers show that the stone diagnosis on CT image is a challenging task while performing segmentation because of the complex structure of interest in the abdomen CT.Therefore, the pre-processing algorithm plays an essential role in improvising the performance of $3 \mathrm{D}$ image segmentation. Along with these techniques, algorithms have been developed for the removal of soft organs and bony skeleton present in the Abdominal CT image (Figure 4). The figure shows the presence of stone in the kidney region.

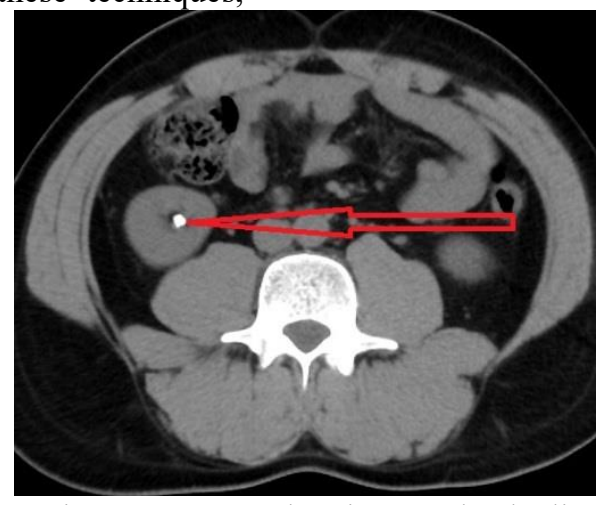

Fig4: CT Image showing renal calculi (Source: Newyork Urology Specialist)

\begin{tabular}{|c|c|c|c|}
\hline Year & Authors & Methods & Performance Measures \\
\hline 2015 & $\begin{array}{c}\text { SamanEbrahimi\& } \\
\text { Vladimir Y } \\
\text { Mariano[12] }\end{array}$ & $\begin{array}{c}\text { Thresholding, } \\
\text { Boundary defining }\end{array}$ & Accuracy: $84.61 \%$ \\
\hline 2018 & $\begin{array}{c}\text { NilarThein et al } \\
{[15]}\end{array}$ & $\begin{array}{l}\text { Three Thresholding } \\
\text { algorithms based on } \\
\text { Intensity, Size \& } \\
\text { Location }\end{array}$ & Sensitivity:95.25\% \\
\hline 2018 & $\begin{array}{l}\text { L Prisilla et al } \\
\text { [24] }\end{array}$ & $\begin{array}{l}\text { Contrast limited } \\
\text { adaptive histogram } \\
\text { equalization }\end{array}$ & Accuracy:87.41\% \\
\hline 2020 & $\begin{array}{c}\text { John Brandon } \\
\text { Graham-Knight et al } \\
{[25]}\end{array}$ & $\begin{array}{c}\text { Kidney } \\
\text { Segmentation using } \\
\text { deep transfer } \\
\text { learning model } \\
\text { nnU-net }\end{array}$ & Dice scores*: 0.9620 \\
\hline 2020 & $\begin{array}{c}\text { Riya Mishr etal } \\
{[23]}\end{array}$ & $\begin{array}{l}\text { Classification using } \\
\text { Back Propagation } \\
\text { Network, Fuzy C- } \\
\text { Mean clustering } \\
\text { algorithm for } \\
\text { segmentation }\end{array}$ & Accuracy:98.8\% \\
\hline
\end{tabular}

* Dice scores is a kind of metrics for evaluating the segmentation of image

Table2: Performance metrics for CT Imaging

\section{Soft Computing and Artificial Intelligence algorithms in Nephrolithiasis Detection}

Soft Computing and Artificial Intelligence algorithms are increasingly employed in Nephrolithiasis detection. Verma J et al (2017) have used the soft computing-based classification techniques $\mathrm{K}$ - Nearest neighbour (KNN) and Support Vector Machine (SVM for the analysis of Nephrolithiasis images [18]. Artificial intelligence (AI) algorithms can be used to accurately detect Nephrolithiasis detection. Considering artificial intelligence methodologies, the neural network provides 
more than $90 \%$ accuracy for both types of detections of Nephrolithiasis detection. Deep Learning Technology is a concept of Machine learning which plays a vital role in medical imaging diagnosis. Convolution Neural Networks (CNN) is one of the deep learning which can be used to classifying detected kidney diseases. CNN architecture is capable of processing a large number of hidden layers as compared to the conventional layered architecture. Kristian M et al (2020) have used Deep Convolution Neural Networks to detect Nephrolithiasis from digital images with better performance [19].G Sumana et al proposed a model which uses Artificial Neural Network as the classifier for diagnosis, GLCM algorithm for feature and Forward Back propagation algorithm to reduce the diagnosis time and increase the accuracy of the model [29]. For Nephrolithiasis detection and analysis CNN, SVM and ANN are most commonly used machine learning algorithms.

- Support Vector Machine: It was introduced in the year 1992, is a supervised learning network which works as a classifier by analysing the data, recognizing pattern and solves linear and non linear classification problems.SVM is considered as a non probabilistic binary linear classifier which takes input pattern as feature vector and identifies to which class the dataset belongs [31]. The figure 5 shows the architecture of the SVM [32].

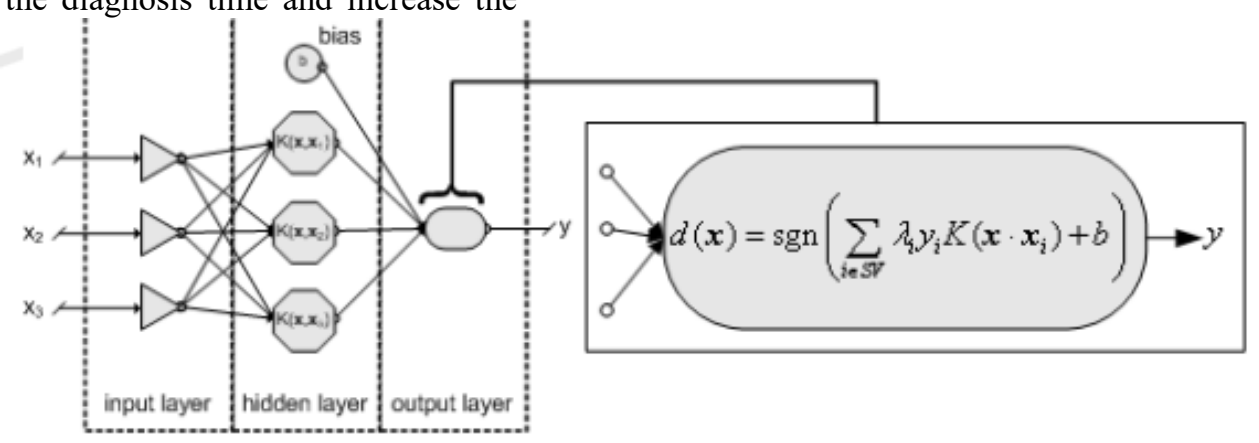

Fig5: Architecture of SVM

- Artificial Neural Network: It's a neural network model and data processing technique that is based on our human brain more like the neuron pathway or the nervous system route. ANN is a network of the processing elements (biological neurons) which work in a parallel manner. The network has input layer (first layer), extra layers

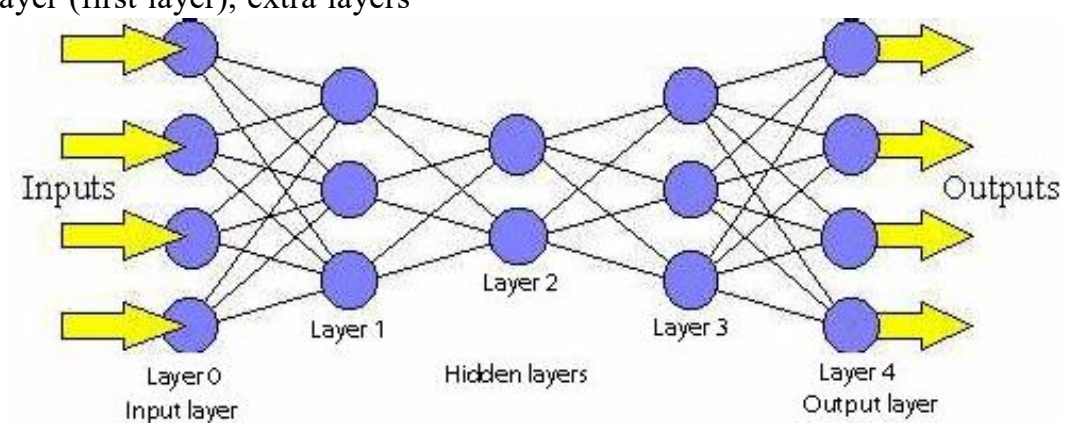

Fig6: ANN Architecture

- Convolution Neural Network: It is a deep learning algorithm which takes images as the input and is capable of differentiating the images from one another by passing it through several layers of network. These layers include Convolution layer and the pooling layer. The convolution layer performs the convolution operation which extracts low level features as in between known as the hidden layer and final layer is the output layer. The main advantage of neural network is that it works swiftly even when there is high noise present in the dataset [29].The basic architecture of the ANN is shown in figure 6 [30]. 


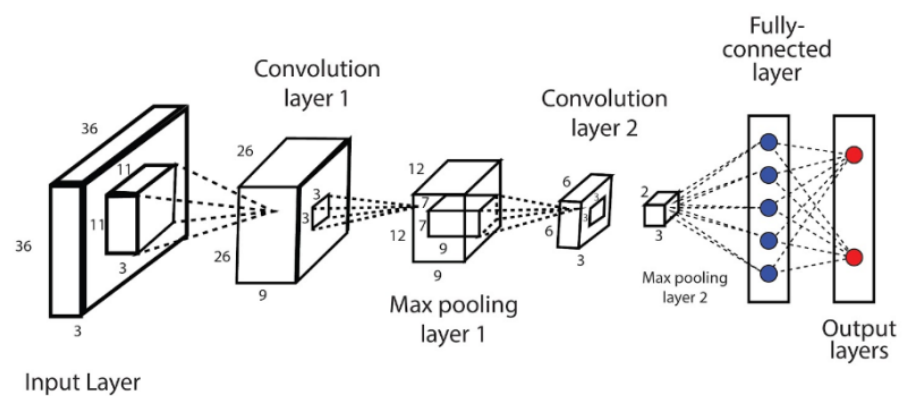

Fig7: CNN Architecture

D. Comparative analysis of the performance of various Nephrolithiasis detection techniques Comparative analysis of the performance and Radiation exposure of various techniques in the diagnosis of
Nephrolithiasis (Fulgham PF et al) such as KUB, Lowdose CT, Ultrasound Imaging, Intravenous pyelography, CT and MRI are presented in Table $3[17,20]$.

\begin{tabular}{cccc}
\hline Modality & Sensitivity & Specificity & $\begin{array}{c}\text { Radiation Exposure in } \\
\text { millisieverts (mSv)[17] }\end{array}$ \\
\hline Low-dose CT & $95 \%[26]$ & $97 \%[26]$ & $\sim 3$ \\
Ultrasound & $61 \%$ & $97 \%$ & None \\
Intravenous pyelogram(IVP) & $70 \%$ & $95 \%$ & 3.0 \\
MRI & $82 \%$ & $98.3 \%$ & None \\
CT & $98 \%$ & $97 \%$ & 10.0 \\
KUB & $57 \%$ & $76 \%$ & 0.7 \\
\hline
\end{tabular}

Table3: Comparative analysis of the performance of various Nephrolithiasis detection technique

\section{Proposed Approach}

An approach is proposed for imaging the subjects with renal stones and an Artificial Intelligence based algorithms and models for processing the Ultrasound or CT scans in order to increase the efficiency and accuracy of diagnosis/prognosis of kidney stones and also to aid the physicians and medical practioners in their decision management for treatment and medications. The initial step is decision making for choosing the right imaging modality based on the clinical symptoms of renal stone, patient's age, health condition of patient that is if she is pregnant or not. For the patients suffering from Nephrolithiasis under the age of 12 and pregnant women, the Ultrasound is to be chosen as the diagnostic modality. Ultrasound imaging modality is used as there is no risk of radiation exposure and the diagnosis can be carried out safely for pregnant woman and children under 12 . The Computed Tomography imaging modality is to be chosen for patients above the age of 12 .CT involves the standard radiation exposure of $10 \mathrm{mSv}$. However, the radiation dose can be modulated as per the requirement and health conditions of the individual. The Low-dose CT has radiation exposure less than $3 \mathrm{mSv}$, but the disadvantage of this is that it results in low quality and resolution. Low quality and poor resolution of the scans obtained from Low-dose CT can be resolved by processing the scans using deep learning techniques. From the research works, the CNN seems promising in improving the quality of the images. This basically involves using two CNNs, in which one network trains and targets the areas of kidney and the other CNN network targets the non-kidney area, and then combining the two networks to generate clean, high quality and resolution and also increases the processing time.

The images obtained from the Ultrasound and CT is uploaded to the computer system for processing. The first step of the processing of image is removing the speckle noise. The filters mentioned in Section III A are effective in removing the speckle noise from the image. If there is no speckle noise present, the image is sent for the next step that is Segmentation and if there is noise present then based on the speckle noise characteristics any of the filter mentioned in Section III A can be used to clean the image. For Image Segmentation process, the CNN with 3D U-net architecture is considered to be best. The U-net architecture is capable of working with few training datasets and samples and can segment images with better efficiency and accuracy. The efficiency of the segmentation process can be checked using the performance parameters such as dice similarity coefficient and weighted loss function (dice loss). Once the image is segmented, it is then passed to the neural network model which is combination of ANN and SVM for feature extraction and classification of the segmented images. The algorithm designed by Priyanka Chak et al is promising in better feature extraction and classification, in which ANN designed, has 12 input nodes, 10 hidden nodes and 2 output nodes and a combination with SVM 
gives accuracy of $99 \%$ with all the features [27]. This algorithm can be used for the feature extraction and segmentation process. The features such as stone location, stone size, stone attenuation and distance between skin and stone extracted from the neural network model will aid the Physician in understanding the Nephrolithiasis condition of the patient better. Further, the features extracted can be combined to statistical Bayesian interface to obtain the probability rank differential diagnosis for further analysis, comparison and study. The basic architecture and brief details of the neural network models and deep learning models proposed in the approach is discussed in section III C. The results obtained can be stored in a database for future reference and study. The flowchart (figure 8) gives an insight of the proposed AI-based system discussed above.

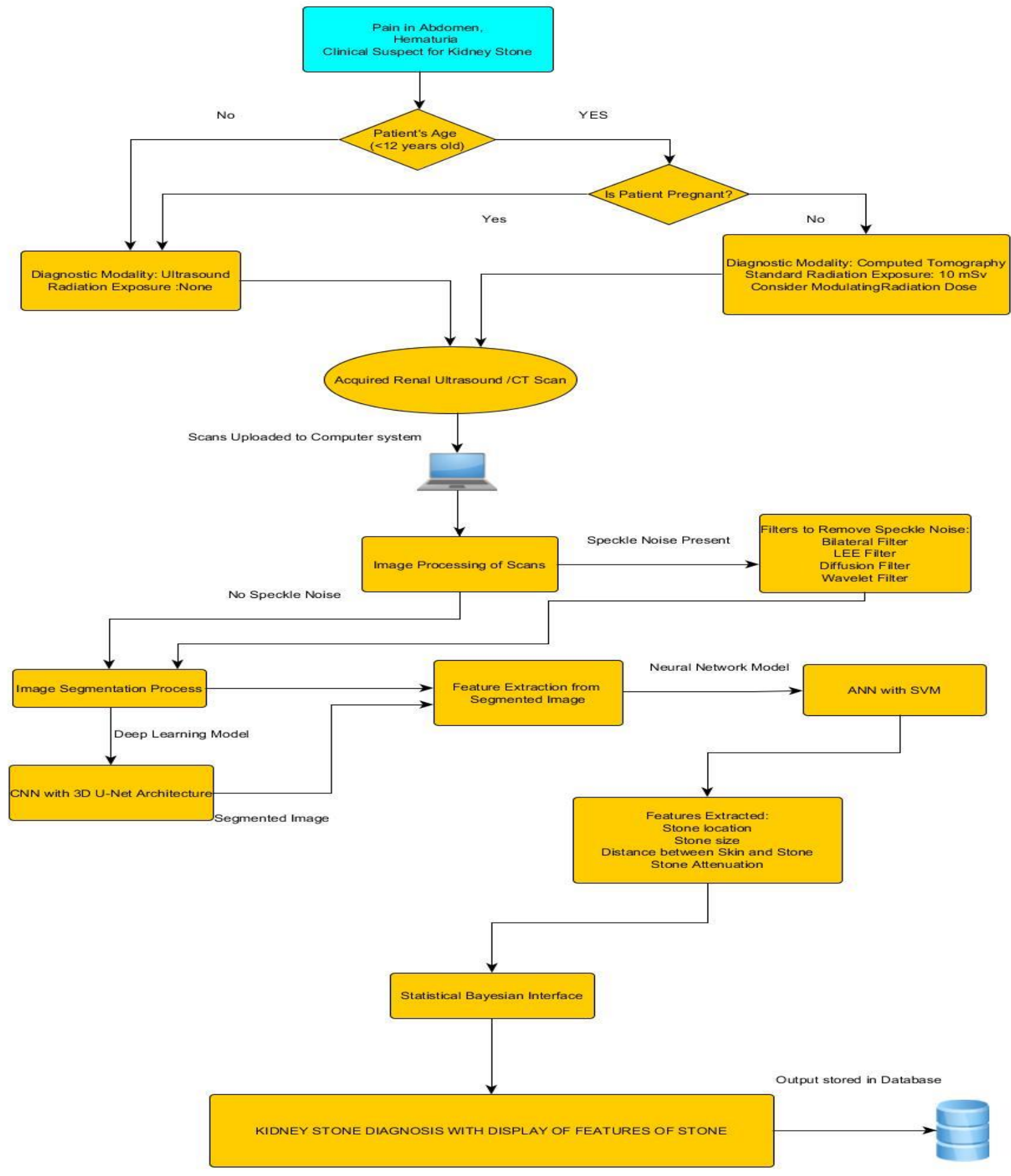

Fig8: Proposed AI-Based System for Detection of Nephrolithiasis

\section{DISCUSSION}

An analysis of various techniques and algorithms that have been employed to obtain better image quality, better de-speckling, improvisation in image contrast, and faster segmentation of the image has been carried out. A comparative analysis of research works on CT and Ultrasound images has also been carried out.
Comparative analysis of the performance of various techniques for the diagnosis of Nephrolithiasis is presented. It is inferred that direct detection of most stones is not possible with MRI. MRIs are generally more expensive than other techniques, such as CT scans. But, MRI produces no dangerous radiation and it can readily reveal urinary obstruction. It has been learnt that both CT imaging and ultrasound imaging techniques detect the 
majority of Nephrolithiasis conditions.In future, there may be a decision support system utilizing the concepts of deep learning for easy and faster processing of the

\section{CONCLUSION}

It has been learnt that Kidney stone detection using Ultrasound Imaging is independent of the composition of stones. This technique is capable of identifying uric-acid stones as well as calcium stones. In the case of Ultrasound imaging, it is not always possible to detect small stones. Unlike ultrasound, CT exposes patients to significant amounts of radiation. The low dose-CT in conjugation with deep learning technique can help improve the quality and resolution of the scan. The ultrasound imaging technique is favoured in the case of pregnant women and people sensitive to IV contrast. Hence, it is recommended that Ultrasounds have to be used for preliminary diagnosis subsequently through CT if the medical practitioner senses it is necessary.

Advances in CT, ultrasonography, KUB radiography and MRI technologies are continuing and are likely to improve all modalities in the future. At present Artificial Intelligence based algorithms are evolving which could improve efficiency. The present trend is the usage of deep learning algorithms for radiographic and sonogram images for Nephrolithiasis detection. The proposed AI based technique discussed in the section 4 when implemented will be promising for faster detection and analysis of kidney stone and serve as the decision support system for the Physicians as well as the radiologists.

In future deep learning can be employed for detecting kidney stone composition during endoscopy. This paves the way to integrated endoscopic and laser systems that automatically provide laser settings based on stone composition recognition which could improve surgical efficiency.Soon, algorithms may be developed to assist in deciding on the best approach for imaging patients.

\section{REFERENCES}

[1]David T. Tzou, ManintUsawachintachit, Kazumi Taguchi, and Thomas Chi, "Ultrasound Use in Urinary Stones: Adapting Old Technology for a Modern-Day Disease", Journal of Endourology, Vol. 31, 2017.

[2] Tamilselvi P R andThangaraj P, "Computer Aided Diagnosis System for Stone Detection and Early Detection of Kidney Stones," Journal of Computer Science, vol.7, no.2, pp.250-254, 2011.

[3] Cunitz B, Dunmire B, Paun M, Sapozhnikov O, Kucewicz J, Hsi R, Lee F, Sorensen M, Harper J and Bailey M, "Improved detection of kidney stones using an optimized Doppler imaging sequence," 2014 IEEE International Ultrasonics Symposium Proceedings, Chicago, United States, pp. 452-455, 2014.

[4] Viswanath $\mathrm{K}$ andGunasundari $\mathrm{R}$, "Design and analysis performance of Kidney Stone Detection from Ultrasound Image by Level Set Segmentation and ANN Classification," 2014 International Conference on Advances in Computing, Communications and Informatics, India, pp. 407-414, 2014.

[5] Vaish P, Bharath R, Rajalakshmi P and Desai U B, "Smartphone based automatic abnormality detection of kidney in ultrasound images," 2016 IEEE 18th International Conference on e-Health Networking, image thereby enhancing the diagnostic efficiency of Nephrolithiasis detection on CT and Ultrasound Images[21]

Applications and Services, Munich, Germany, pp.1-6, 2016

[6] Verma J, Nath M, Tripathi P andSaini KK, "Analysis and identification of kidney stone using Kth nearest neighbour (KNN) and support vector machine (SVM) classification technique," Pattern Recognition and Image Analysis, vol.27, no.3, pp.574-580, 2017.

[7] S. M. K. Chaitanyaand P. Rajesh Kumar, "Classification of Kidney Images Using Cuckoo Search Algorithm and Artificial Neural Network", International Journal of Engineering and Advanced Technology, Vol.8, No.3, 2019.

[8] D. Y. Kim and J. W. Park, "Computer-Aided detection of kidney tumor on abdominal computed tomography scans", ActaRadiologica, vol. 45, no. 7, pp. 791-795, 2004

[9] J. Kolomaznık, "Fast Segmentation of Kidneys in CT Images, Proceedings of Contributed Papers", Part I, pp.70-75, 2010.

[10] $\mathrm{Wu}$ Zhou and YaoqinXie, "Interactive Medical Image Segmentation Using Snake and Multiscale Curve Editing", Hindawi Mathematical Methods and Applications in Medical Imaging, Vol. 2013, pp.1-13.

[11] Liu, J., Wang, S., Turkbey, E. B., Linguraru, M. G., Yao, J., andSummers, R. M., "Computer-aided detection of renal calculi from non- contrast CT images using TVflow and MSER features", Medical Physics, Vol.42, No.1, pp.144-153, 2014.

[12] SamanEbrahimi and Vladimir Y. Mariano, "Image Quality Improvement in Kidney Stone Detection on Computed Tomography Images, Journal of Image and Graphics", Vol. 3, No. 1, 2015.

[13] SujataNavratnam, SitiFazilahValliappan Raman and SundaresanPerumal."Seed Pixel Region Growing Segmentation and Artificial Neural Network Classifier for Detecting the Renal Calculi in Ultrasound Images for Urologist Decisions", International Journal of Computer Science Issues, Vol. 13, No. 5, pp. 1694-0814, 2016.

[14] Akkasaligar, P. T., Biradar, S. and Kumbar, V. "Kidney stone detection in computed tomography images", Proceedings of the International Conference on Smart Technologies For Smart Nation, 2017.

[15] Thein N, Nugroho H A, Adji T B andHamamoto K, "An image preprocessing method for kidney stone segmentation in CT scan images," International Conference on Computer Engineering, Network and Intelligent Multimedia, Surabaya, Indonesia, pp.147-150, 2018.

[16] Stalina S, Aditi S, Anuja R and Prof. Pooja L Gohel, "Kidney Stone Detection Using Image Processing On CT Images," International Journal of Management, Technology And Engineering, vol.8, no.9, pp. 2257-2260, 2018.

[17] Fulgham PF, Assimos DG, Pearle MS, and Preminger GM. "Clinical effectiveness protocols for imaging in the management of ureteral calculous disease: AUA technology assessment", J Urol. 2013;189:12031213. 
[18] Verma, J., Nath, M., Tripathi, P, “ Analysis and identification of kidney stone using Kth nearest neighbour (KNN) and support vector machine (SVM) classification techniques", Pattern Recognit. Image Anal. 27, 574-580 (2017).

[19] Kristian M. Black Hei Law Ali AldoukhiJia Deng Khurshid R. Ghani, "Deep learning computer vision algorithm for detecting kidney stone composition", BJU International, Volume125, Issue 6, 2020, pp. 920-924.

[20] Wayne Brisbane, Michael R. Bailey and Mathew D. Sorensen, "An overview of kidney stone imaging techniques", Nat Rev Urol. 2016 November; 13(11): 654662.

[21] Saxena, Ashish, Eddie Yin Kwee Ng, and SooTeik Lim. "Imaging modalities to diagnose carotid artery stenosis: progress and prospect", Biomedical engineering online 18.1 (2019): 66.

[22] Cincotti G, Loi G, Pappalardo M. Frequency decomposition compounding of ultra-sound medical images with wavelet packets. IEEE Trans Med Imag 2001;20(8):764-71.

[23] Riya Mishr,Avik Bhattacharjee,M.Gayathri, C.Malathy,Kidney Stone Detection with CT Images Using Neural Network,International Journal of Psyshosocial Rehabilitation,Vol.24,Issue 08,2020

[24] L. Prisilla ,I. Laurance Aroqiaraj, Kidney Stone Detection using Contrast Limited Adaptive Histogram Equalization (CLAHE) on CT Scan Images, International Journal of Computational Intelligence and Informatics, Vol. 7: No. 4, March 2018

[25] Graham-Knight J.B. et al. (2020) Accurate Kidney Segmentation in CT Scans Using Deep Transfer Learning. In: McDaniel T., Berretti S., Curcio I., Basu A. (eds) Smart Multimedia. ICSM 2019. Lecture Notes in Computer Science, vol 12015. Springer, Cham.
[26] Coursey CA, et al. ACR Appropriateness Criteria(R) acute onset flank pain-suspicion of stone disease. Ultrasound Q. 2012;28:227-

233. [PubMed] [Google Scholar]

[27] Chak P., Navadiya P., Parikh B., Pathak K.C. (2020) Neural Network and SVM Based Kidney Stone Based Medical Image Classification. In: Nain N., Vipparthi S., Raman B. (eds) Computer Vision and Image Processing. CVIP 2019.Communications in Computer and Information Science, vol 1147. Springer, Singapore.

[28] https://www.webmd.com/kidneystones/understanding-kidney-stones-basics\#1

[29] G. Sumana ,G. Anjan Babu, Prediction of Nephrolithiasis Based on Extracted Features of CT-Scan Images using Artificial Neural Networks, International Journal of Advanced Research in Computer Science, Volume 8, No. 5, May-June 2017

[30] http://www.neuralforecasting.com/support vector machines.htm [31] Abhishek, Gour Sundar Mitra Thakur, Dolly Gupta, Proposing Efficient Neural Network Training Model for Kidney Stone Diagnosis, (IJCSIT) International Journal of Computer Science and Information Technologies, Vol. 3 (3) , 2012,3900-3904

[32] http://www.neuralforecasting.com/support_vector_machines.htm [33] https://www.analyticssteps.com/blogs/convolutionalneural-network-cnn-\%20graphical-visualization-codeexplanation 